\title{
Incidence of Left Ventricular Systolic Dysfunction in Asymp- tomatic, Incidently Detected Left Bundle Branch Block (LBBB) in Apparently Healthy Individuals: A Prospective Observation- al Study at a Tertiary Care Cardiac Center in North India
}

Sheikh Jan Mohammad, MD, DNB, AM-ACC/AHA ${ }^{1 *}$, Mohammad Tahir Ganaye, MD², Samiera Hassan $^{3}$ and Nusrat Bashir ${ }^{4}$

${ }^{1}$ Consultant Cardiologist, Florence Hospital, Jammu and Kashmir, India

${ }^{2}$ Consultant Physician, Department of Health and Family Welfare, Jammu and Kashmir, India

${ }^{3}$ Medical Officer, Department of Preventive and Social Medicine, Government Medical College, Jammu and Kashmir, India

${ }^{4}$ BUMS Intern, ASYM District Hospital, Jammu and Kashmir, India

*Corresponding author: Dr. Sheikh Jan Mohammad, MD, DNB, AM-ACC/AHA, Consultant Cardiologist, Florence Hospital, Jammu and Kashmir, 191113, India, Tel: +919599772219

\begin{abstract}
Background: Left bundle branch block (LBBB) is often associated with significant heart disease and is often the result of myocardial injury, strain or hypertrophy, it can also be seen in patients without any particular clinical disease. The significance of LBBB has not been well studied in subjects without myocardial infarction or pre-existing heart failure. Therefore, we sought to study the incidence of LV systolic dysfunction in asymptomatic, incidentally detected LBBB in apparently healthy individuals.
\end{abstract}

Aims and objectives: To study the incidence of LV systolic dysfunction in asymptomatic incidentally detected LBBB in apparently healthy individuals.

Material and methods: A total of 150 patients were enrolled for study over a period of two years after written informed consent was obtained. All patients included in study attended hospital outpatient department for general and routine check-up and where completely asymptomatic. Patients with incidentally detected LBBB on 12 lead surface Electrocardiogram (ECG) were further evaluated by Echocardiography for LV systolic function.

Results: The study observed LBBB is more common in males $(61.3 \%)$ and is very rare below 40 years of age $(0.053 \%)$. LBBB is common clinical entity after 60 years of age $(40 \%$ in our group). Our study found asymptomatic LBBB patients have normal LV systolic function, paradoxical septal motion, normal ejection fraction (EF $>50 \%$ ) in $54 \%$ of patients. Asymptomatic LBBB rarely has more than mild LV systolic dysfunction on echocardiography. We found only $2 \%$ of patients with $\mathrm{EF}<40 \%$ ).

Conclusion: Asymptomatic LBBB is more common in males and is very rare below 40 years of age. Asymptomatic LBBB patients have near normal LV systolic function. Significant numbers of patients with asymptomatic LBBB have subclinical LV systolic dysfunction. Significant LV systolic dysfunction in LBBB is uncommon in asymptomatic patients.

\section{Keywords}

Asymptomatic LBBB, ECG criteria, LV dysfunction, Echo in LBBB, Criteria for LBBB

\section{Introduction}

Left bundle branch block (LBBB) is often associated with significant heart disease and is often the result of myocardial injury, strain or hypertrophy, it can also be seen in patients without any particular clinical

Citation: Mohammad SJ, Ganaye MT, Hassan S, Bashir N (2020) Incidence of Left Ventricular Systolic Dysfunction in Asymptomatic, Incidently Detected Left Bundle Branch Block (LBBB) in Apparently Healthy Individuals: A Prospective Observational Study at a Tertiary Care Cardiac Center in North India. Int J Clin Cardiol 7:211. doi.org/10.23937/2378-2951/1410211

Accepted: December 29, 2020: Published: December 31, 2020

Copyright: (c) 2020 Mohammad SJ, et al. This is an open-access article distributed under the terms of the Creative Commons Attribution License, which permits unrestricted use, distribution, and reproduction in any medium, provided the original author and source are credited. 
disease. In isolation the presence of LBBB does not lend itself to any specific clinical concern, nor does it affect prognosis. However, in the proper clinical context, LBBB can of great consequence and importance, especially in patients presenting with acute chest pain, syncope and in those suffering from heart failure with reduced ejection fraction (HFrEF) [1]. New onset LBBB in the proper setting of concerning clinical symptoms should always be considered a sign of pathology and can indicate myocardial infarction. New LBBB is considered an ST-segment elevation equivalent in patients presenting with chest pain [2]. Although the QRS and ST segments of an ECG are traditionally regarded as un-interpretable in the presence of LBBB, emerging Sgarbossa criteria have been developed allowing some interpretation of ECGs despite LBBB $[3,4]$. The ECG criteria for a left bundle branch block include:

1. QRS duration greater than 120 milliseconds.

2. Absence of $Q$ wave in leads I, V5 and V6.

3. Monomorphic R wave in I, V5 and V6.

4. ST and T wave displacement opposite to the major deflection of the QRS complex.

A simple way to diagnose a left bundle branch in an ECG with a widened QRS complex (>120 ms) would be to look at lead V1. If the QRS complex is widened and downwardly deflected in lead V1, a left bundle branch block is present. If the QRS complex is widened and upwardly deflected in lead V1, a right bundle branch block is present. The image below shows the typical findings of a left bundle branch block in the precordial ECG leads. LBBB is prevalent in about $0.06 \%$ to $0.1 \%$ of the general population. Approximately $33 \%$ of patients with heart failure have LBBB. Incidence increases with severity of left ventricular failure in heart failure patients [1]. LBBB itself is asymptomatic, there are no signs or symptoms other than the distinct pattern on EKG $[1,5]$. LBBB is detected on ECG. Diagnostic criteria are defined by the American College of Cardiology (ACC) and American Heart Association (AHA) as follows:

1. Rhythm must be of super-ventricular origin (EG: Ventricular activation coming from atrial or AV nodal activation).

2. QRS duration greater than $120 \mathrm{~ms}$.

3. Lead V1 should have either a QS or a small $r$ wave with large $S$ wave.

4. Lead V6 should have a notched $R$ wave and no $Q$ wave [6].

In the setting of acute myocardial infarction, there are a set of criteria called Sgarbossa criteria which can be applied to the ECG to increase predictive value for or against myocardial infarction. These criteria are not as good as ST-segment elevation in the absence of LBBB. Their sensitivity is only $49 \%$, but specificity is greater than $90 \%$.

1. Concordant ST elevation greater than $1 \mathrm{~mm}$ in leads with a positive QRS complex (5 points).

2. Concordant ST depression greater than $1 \mathrm{~mm}$ in V1 to V3 (3 points).

3. Discordant ST elevation greater than $5 \mathrm{~mm}$ in leads in a negative QRS complex (2 points).

Three or more points means acute myocardial infarction.

Modified Sgarbossa criteria were validated in 2015. The sensitivity of the modified criteria increases to $80 \%$ without affecting specificity. The third criteria regarding greater than $5 \mathrm{~mm}$ of discordance were chosen rather arbitrarily. The modified criteria change $5 \mathrm{~mm}$ to greater than $25 \%$ of the downward QRS deflection. Criteria 3 is modified as follows: Discordant ST elevation greater than $25 \%$ of downward QRS deflection in a negative QRS complex (2 points).

Differentials for LBBB include intra-ventricular conduction delay - which can result in similar ECG findings but often will not have an $R$ wave in V6. A paced rhythm can often be confused with LBBB but again often does not have an $R$ wave in $V 6$ and pacer spikes are usually seen. An LBBB pattern with QRS duration less than 120 $\mathrm{ms}$ is called an incomplete LBBB. A ventricular rhythm (run of PVC's) without super-ventricular stimulation can appear very similar to a left bundle branch block and in certain uncommon situations would be indistinguishable from LBBB [6].

In healthy individuals, LBBB does not confer any specific or additional risk. Mortality hazard ratio (HR) for LBBB is only 1.3 of normal. However, in patients with new onset, LBBB mortality HR is greater than 10-times normal [1]. Specific at-risk populations of patients with $\angle B B B$ are those who are presenting with chest pain and have new-onset LBBB which should be considered equivalent to ST-segment elevation [2]. In patients with heart failure, the presence of left bundle branch block is associated with increased cardiovascular outcomes and mortality. However, in a recent study which attempted to isolate LBBB's sole contribution to outcomes found that if contributions of confounders are excluded LBBB contributes far more modestly to poor outcomes. This is likely because LBBB is more of a symptom of dilated cardiomyopathy as opposed to a causative agent in the progression of the disease [7]. The significance of LBBB has not been well studied in subjects without myocardial infarction or pre-existing heart failure. Therefore, we sought to study the incidence of LV systolic dysfunction in asymptomatic, incidentally detected LBBB in apparently healthy individuals.

\section{Aims and Objectives}

To study the incidence of LV systolic dysfunction in 
asymptomatic incidentally detected LBBB in apparently healthy individuals.

\section{Material and Methods}

Study was conducted at a tertiary care cardiac center, 150 patients were enrolled for study over a period of two years. A written informed consent was obtained from all patients. All patients who were enrolled for study attended hospital outpatient department for general and routine assessment and were found completely asymptomatic at presentation. Patients with incidently detected LBBB on 12 lead surface Electrocardiogram (ECG) were further evaluated by Echocardiography for LV systolic function.LBBB was defined as 1) QRS duration $>120 \mathrm{~ms}, 2$ ) $P Q$ interval $>120 \mathrm{~ms}$, 3) Predominantly upright complexes with slurred $R$ waves in leads $I, V_{5^{\prime}}$ and $V_{6}$, and 4) Small of absent $R$ waves in leads $V_{1}$ and $V_{2}$. Complete left ventricular Echocardiographic systolic function analysis included M-mode, wall motion abnormality, fractional area change (FAC), fractional shortening, paradoxical septal motion defects, Tissue Doppler imaging (TDI) and stress strain imaging. Ejection fraction was calculated by M-mode, eye balling and Simpson's method of disk.

\section{Inclusion criteria}

1. Age $>18$ years.

2. Asymptomatic adults.

3. ECG criteria for LBBB.

4. No known cardiac illness.

\section{Exclusion criteria}

1. Symptomatic patients with LBBB.

2. Known cardiac or non-cardiac illness on follow up.

3. Post-CPR revived patients.

4. Consent not given for study

\section{Global left ventricle systolic function}

Maximum rate of change of pressure $(\mathrm{dP} / \mathrm{dt}$ max) during the systole may be an appropriate measure but this requires an intramyocardial or intracavity micromanometer, and since these laboratory modalities cannot be used routinely in clinical scenario, noninvasive techniques which measure a mean pressure change or a change in volume or other surrogates of volume become handy. Global LV systolic function can be indirectly assessed by echocardiography using the following indications:

- Changes in LV volume or LV dimension, including cardiac output (CO).

- Systolic index of contractility (dP/dt).

- Global longitudinal strain (GLS) with speckle tracking echocardiography (STE).

\section{Changes in the left ventricle volumes and dimen- sions}

Two dimensional echocardiography, 2D-guided M-mode echocardiography, and Doppler echocardiography are used to measure the dimensions and volume of LV cavity. Recommendation for the measurements using $\mathrm{M}$-mode is from the leading edge to leading edge while that for $2 D$ is from trailing edge to leading edge. Global LV systolic function can be assessed using changes in the LV dimensions and volumes between LV diastole and systole. The recommended calculations are as follows:

- Fractional shortening (FS)

- Fractional area change (FAC)

- Ejection fraction (EF)

- Stroke volume (SV) and CO

\section{Fractional shortening}

The measurement of LV diameter taken just below or at the tip of the mitral valve leaflets in the TG 2 C view or LV diameter taken exactly through the center point of the LV cavity in SAX views, either basal or mid-papillary level, during diastole and systole will help us to calculate FS of LV at that plane by the: FS = LVIDd-LVIDs/ LVIDd $\times 100 \%$. Where, LVIDd $=$ LV internal diameter at end diastole and LVIDs = LV internal diameter at end systole. In M-mode echocardiography, aligning M-mode cursor just at the tip of mitral leaflets or exactly perpendicular to the inferior wall and passing through the center of the LV cavity will give us a M-mode trace, and these measurements can be timed more accurately. Since mechanical systole lags behind the electrical systole, the measurement of $L V$ dimension when the time cursor is placed at or immediately before the peak of the R-wave in QRS complex is considered as LVIDd, and the $\mathrm{LV}$ dimension at the end of T-wave in electrocardiogram (ECG) is taken for LVIDs. This is applicable for all 2D and M-mode measurements (Figure 1).

\section{Biplane Simpson's method for ejection fraction (EF)}

This method is currently recommended for the calculation of LV volumes and EF. This method employs the principle of summation of twenty cylindrical discs of equal height. Diameter of the cylinder will varies depending on the shape of the LV cavity. This requires the LV cavity to imaged in LAX including the base and apex in two orthogonal planes. The endocardial border has to be drawn and connected at the mitral valve level by a straight line. Inbuilt software in all the echocardiography machines would automatically divide this $\mathrm{LV}$ area into twenty equal divisions once the LV LAX has been marked from the apex to the middle of the line joining the mitral annulus. LV volumes are measured at end diastole and end systole in both the planes and 


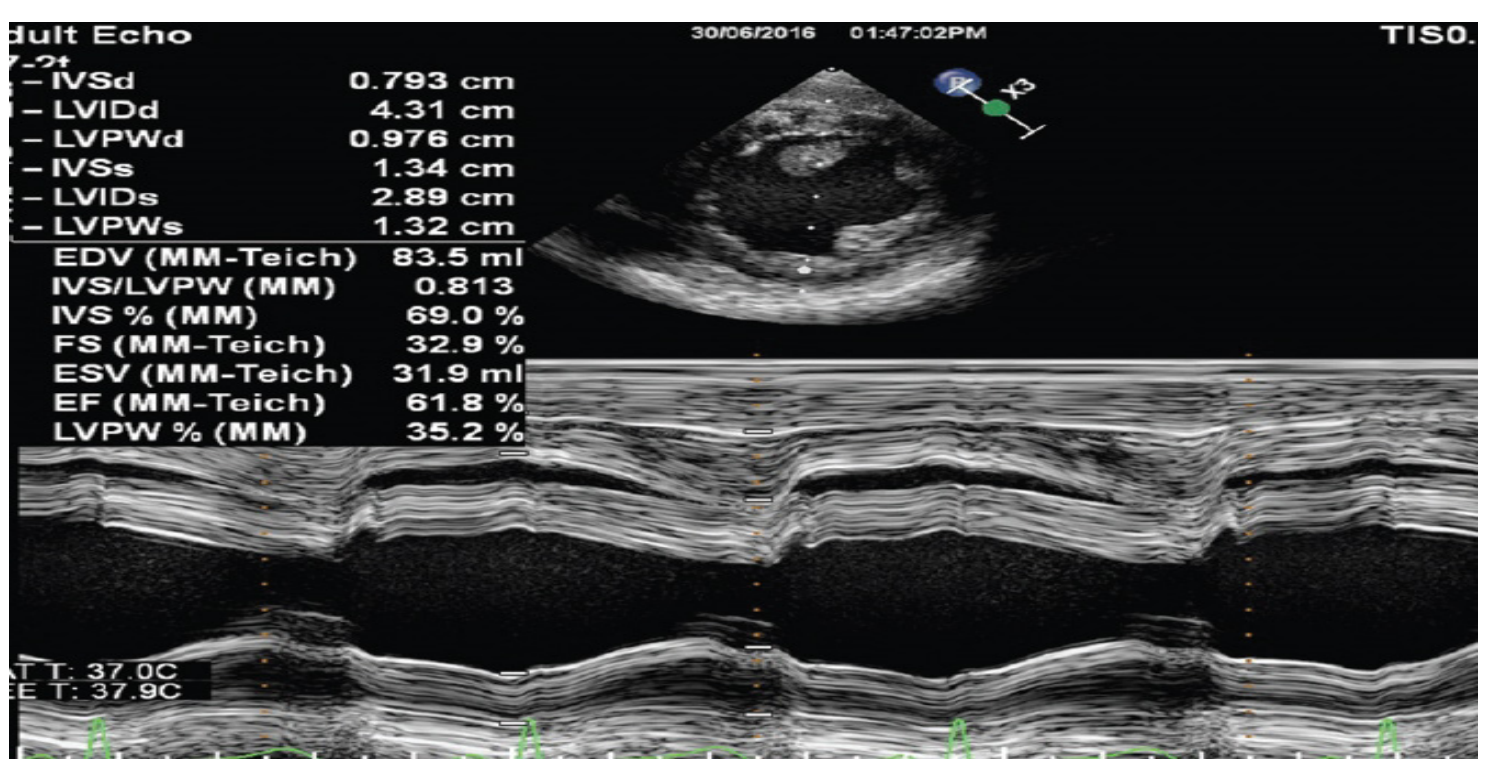

Figure 1: FS (Fractional Shortening) measurement using M-mode.

- Analyse data from patients for routine health
check up
- Exclude all patients not fullfillig inclusion criteria.

used in the equation for calculating the EF.

\section{Reference values:}

- Normal LV function - > 55\%.

- $\quad$ Mild LV dysfunction - 45\%-54\%.

- Moderate LV dysfunction - 30\%-44\%.

- Severe LV dysfunction - < 30\%.

\section{Systolic index of contractility (DP/DT)}

The maximum rate of rise of LV pressure during the isovolumic contraction phase of LV systole, $\mathrm{dP} / \mathrm{dt}$, is a good measure of LV contractility. This is not affected by afterload and very minimally influenced by preload. The prerequisite for this measurement using 2D and
Doppler echocardiography is that the associated mitral valve should have some degree of central mitral valve regurgitation (MR). In the presence of global LV dysfunction, the LV pressure build up will decrease and the LA pressure will increase which will decrease the rate of rise of MR jet velocity. This is the principle behind this method of assessing global LV function.

\section{Reference Values: Changes in dp/dt over time}

\begin{tabular}{|l|l|l|}
\hline $\mathbf{L V}$ function & $\mathbf{d P} / \mathbf{d t}(\mathbf{m m H g} / \mathbf{s})$ & Time $(\boldsymbol{\Delta t})(\mathbf{m s})$ \\
\hline Normal & $>1200$ & $\leq 27$ \\
\hline Borderline & $800-1200$ & \\
\hline Reduced & $<800$ & $\geq 32$ \\
\hline Severely reduced & $<500$ & \\
\hline
\end{tabular}




\section{LV: Left Ventricle}

\section{Statistical Analysis}

Data was tabulated in MS Office Excel worksheet. Descriptive statistics was computed for all the numerical data. Frequency tables were constructed for categorical data. Matrix plots, flow charts and figures were drawn as per need. Data was analysed for incidence of LV systolic dysfunction in asymptomatic incidently detected LBBB in subjects who attended our hospital for routine health check up and for other illness or indications (Figure 2).

\section{Results and Observation}

We enrolled 150 patients for study out of which there were 92 males (61.3\%) and 58 females (38.66\%). We found LBBB is more common in males than females ( $\mathrm{Fi}$ gure 3$)$.

Our study showed LBBB is very rare below 40 years of age $(0.053 \%)$ and common clinical entity after 60 years of age ( $40 \%$ in our group). Probably depicting the various age related etiology (Figure 4).

Our study found asymptomatic LBBB patients have

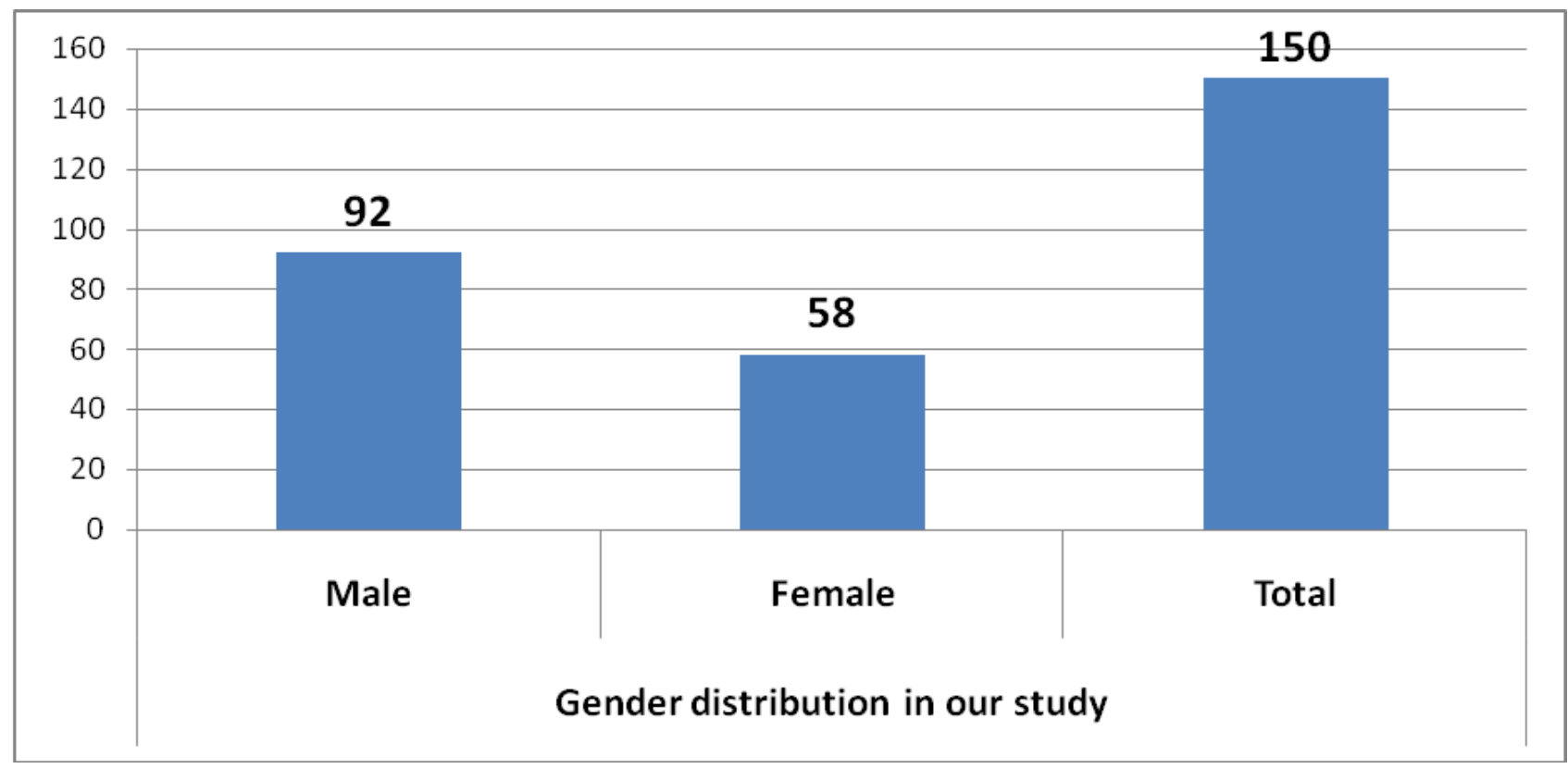

Figure 3: Gender distribution.

\section{Fig-2Distribution of patients in various age Groups}

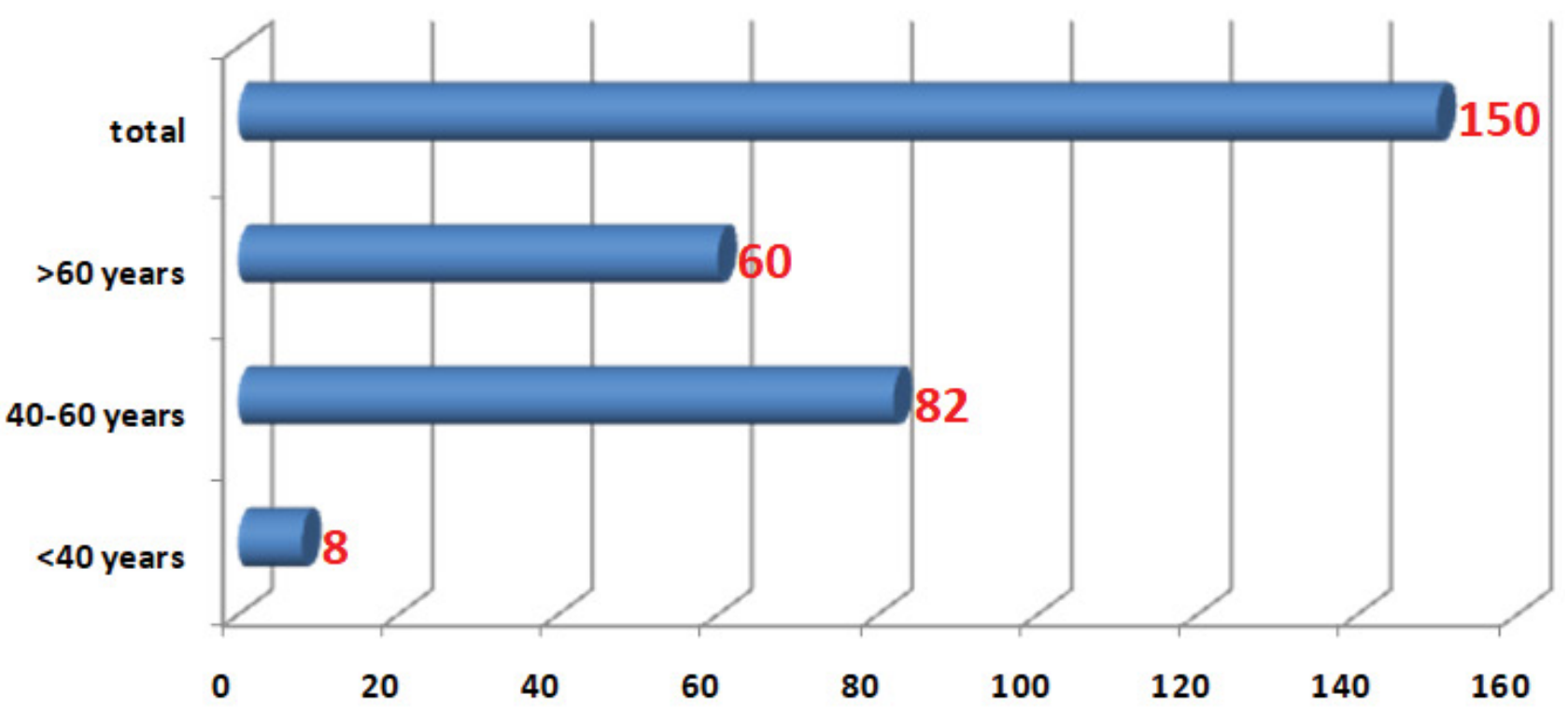

Figure 4: Distribution of patients in various age groups. 


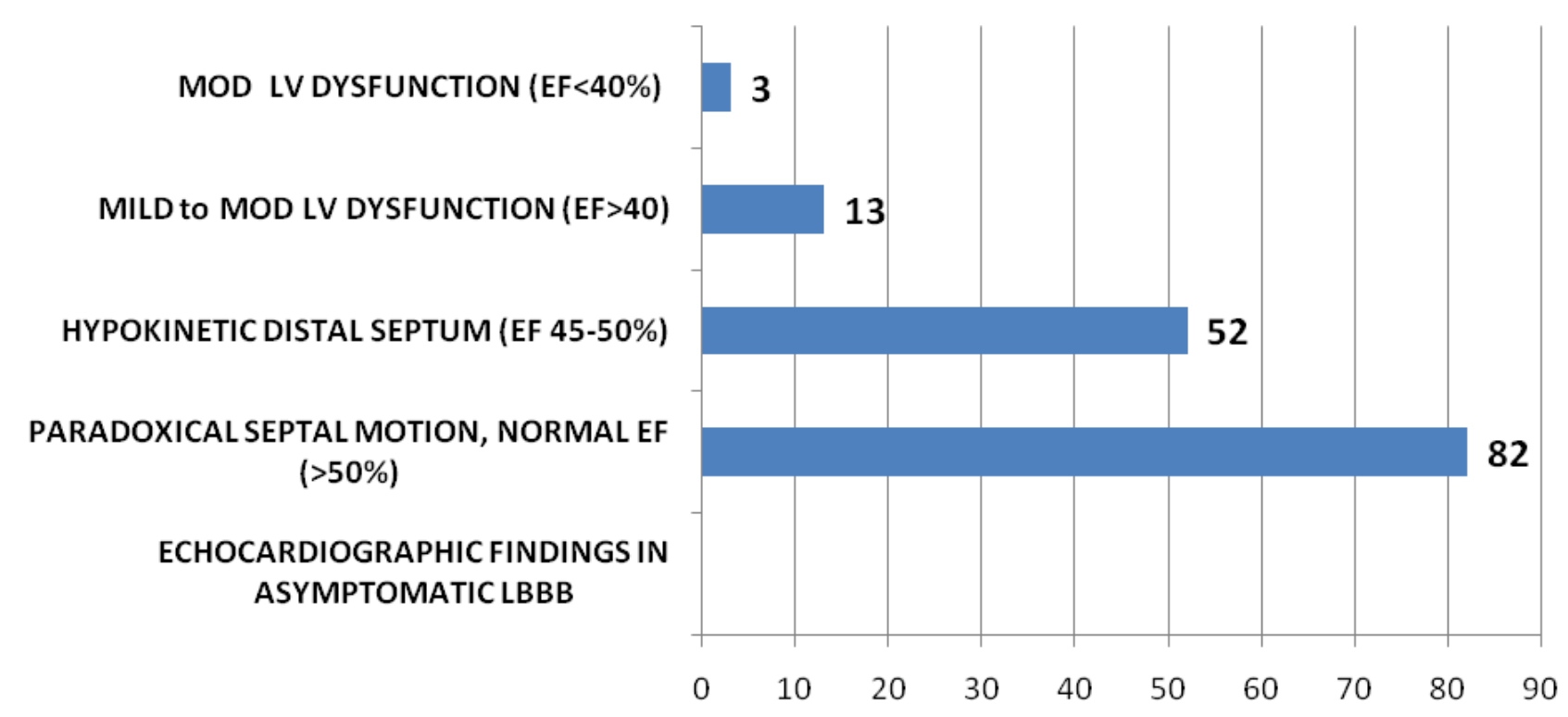

Figure 5: Echocardiographic findings in study.

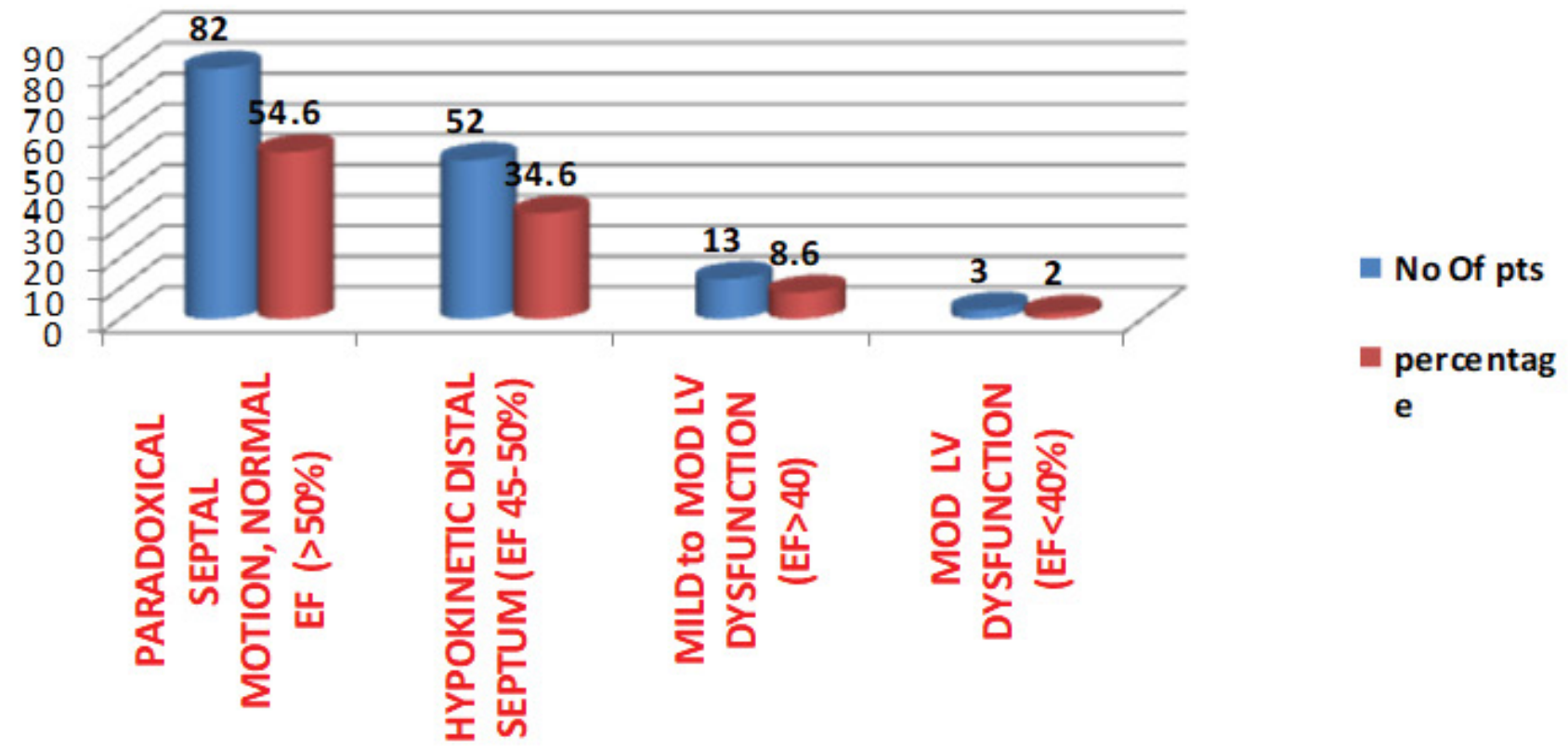

Figure 6: Different echocardiographic findings.

most commonly paradoxical septal motion on echo with normal ejection fraction (EF) (82 patients $\{54 \%\} \mathrm{EF}$ $>50 \%$ ). Asymptomatic LBBB rarely has more than mild LV systolic dysfunction (3 patients $\{2 \%\}$ with $\mathrm{EF}<40 \%$ ) (Figure 5).

We observed $54 \%$ patients have normal Global LV systolic function (LVEF > 50\%) with paradoxical IVS motion only. We observed $34.6 \%$ have minimal LV systolic dysfunction (LVEF $=45-50 \%$ ). It's rare to have significant LV systolic dysfunction only $2 \%$ had moderate LV systolic dysfunction (LVEF $=<40 \%$ ) (Figure 6).

\section{Discussion}

It was prospective observational study for two years at a tertiary care cardiac center in central Kashmir.
We enrolled 150 patients of asymptomatic incidentally detected LBBB detected on routine assessment in our outpatient department. There were 92 males (61.3\%) and 58 females (38.66\%). LBBB is more common in males than females. Study showed LBBB is very rare below 40 years of age $(0.053 \%)$ and common clinical entity after 60 years of age ( $40 \%$ in our group). This indicates various etiology for LBBB such as hypertension, ischemic heart disease, cardiomyopathies and other co-morbidities which are present in older age patients. Our study found majority of asymptomatic LBBB patients have normal LV systolic function, paradoxical septal motion on Echocardiography with normal ejection fraction, 82 patients $\{54 \%\} \mathrm{EF}>50 \%$. Asymptomatic LBBB rarely has more than mild LV systolic dysfunction ( 3 patients $\{2 \%\}$ with $\mathrm{EF}<40 \%)$. Prevalence of $\mathrm{LBBB}$ in the general popu- 
lation has been reported to range from $0.1-0.8 \%$, and there is a large amount of strong data out there about the significance of LBBB on prognosis. Framingham study [8] reported; within 10 years from LBBB detection, CV mortality was $50 \%$; and at 18 years follow-up, only $11 \%$ of LBBB patients remained free of detectable $\mathrm{CV}$ abnormalities. Azadani, et al. [9] in 2012 found that in asymptomatic LBBB patients during 6 years of follow-up, $4.8 \%$ of people developed new CHF. A higher mortality from cardiovascular diseases was also found in participants with LBBB after adjusting for potential confounders.

There are some important questions which need to be answered in apparently healthy individual with asymptomatic LBBB on ECG:

- Do all asymptomatic LBBB patients need further extensive evaluation to look for cause?

- Finding asymptomatic LV systolic dysfunction (LVSD) is important, but is it not more likely they will present with a change in symptoms?

- Should all asymptomatic LBBB patients be treated as new onset LBBB if previous ECG is not available?

- Should all patients go for EP study to detect advanced conduction system disease and need for early intervention?

- If these investigations are normal, after reassuring your patient and counselling on minimising $\mathrm{CV}$ risk factors, what next?

- What should be the follow up of patients with asymptomatic LBBB, those less than and above 50 years of age?

\section{What we suggest}

- Follow them at regular intervals, record baseline relevant investigations including ECG and Echocardiography.

- Periodic echocardiograms to establish they haven't developed sub-clinical LVSD.

- Symptomatic patients definitely need further evaluation.

- Give them a copy of their ECG, to ease their path through future medical contacts, and counsel them to report any symptoms consistent with heart failure, IHD and any syncopal episodes.

- Life style modification to minimise CV risk.

- Last and most importantly we need further studies with large sample size to establish clinical guidelines and risk stratification for these patients.

\section{Conclusion}

- Asymptomatic LBBB is more common in males and is very rare below 40 years of age.
- Asymptomatic LBBB patients have near normal LV systolic function.

- Paradoxical IVS motion is most common echocardiographic finding.

- Significant numbers of patients with asymptomatic LBBB have subclinical LV systolic dysfunction.

- Significant LV systolic dysfunction in LBBB is rarely found in asymptomatic patients.

\section{References}

1. Francia P, Balla C, Paneni F, Volpe M (2007) Left bundle-branch block--pathophysiology, prognosis, and clinical management. Clin Cardiol 30: 110-115.

2. O'Gara PT, Kushner FG, Ascheim DD, Casey Jr DE, Chung MK, et al. (2013) 2013 ACCF/AHA guideline for the management of ST-elevation myocardial infarction: A report of the American College of Cardiology Foundation/American Heart Association Task Force on Practice Guidelines. J Am Coll Cardiol 61: e78-e140.

3. Sgarbossa EB, Pinski SL, Barbagelata A, Underwood DA, Gates KB, et al. (1996) Electrocardiographic diagnosis of evolving acute myocardial infarction in the presence of left bundle-branch block. GUSTO-1 (Global Utilization of Streptokinase and Tissue Plasminogen Activator for Occluded Coronary Arteries) Investigators. N Engl J Med 334: 481487.

4. Meyers HP, Limkakeng Jr AT, Jaffa EJ, Patel A, Theiling BJ, et al. (2015) Validation of the modified Sgarbossa criteria for acute coronary occlusion in the setting of left bundle branch block: A retrospective case-control study. Am Heart J 170: 1255-1264.

5. Clark AL, Goode K, Cleland JGF (2008) The prevalence and incidence of left bundle branch block in ambulant patients with chronic heart failure. Eur J Heart Fail 10: 696702.

6. Nikoo MH, Aslani A, Jorat MV (2013) LBBB: State-of-theart criteria. Int Cardiovasc Res J 7: 39-40.

7. Yancy CW, Jessup M, Bozkurt B, Butler J, Casey Jr DE, et al. (2013) 2013 ACCF/AHA guideline for the management of heart failure: A report of the American College of Cardiology Foundation/American Heart Association Task Force on Practice Guidelines. J Am Coll Cardiol 62: e147-e239.

8. Schneider JF, Thomas HE Jr, Kreger BE, McNamara PM, Kannel WB (1979) Newly acquired left bundle-branch block: The Framingham study. Ann Intern Med 90: 303-310.

9. Azadani PN, Soleimanirahbar A, Marcus GM, Haight TJ, Hollenberg M, et al. (2012) Asymptomatic left bundle branch block predicts new-onset congestive heart failure and death from cardiovascular diseases. Cardiol Res 3: 258263. 